\title{
PICOSECOND LASER PHOTOLYSIS STUDIES UPON PHOTOCHEMICAL ISOMERIZATION AND PROTOLYTIC REACTION OF A STILBAZOLIUM BETAINE
}

\author{
N. IKEDA $¥$, N. MATAGA \\ Deparsment of Chemistry. Faculty of Engineering Science. Osaka University. Toyonaka. Osaka 560, Japan
}

\author{
U. STEINER \\ and \\ M.H. ABDEL-KADER \\ Chemistry. Department, Faculty of Science, Tanta University. Tanta, Egypt
}

Fakultät fïr Chemie. Liniversirät Konstanz. D-7750 Konstanz 1, Federal Republic of Germany'

\begin{abstract}
The dynamical behavior of a stilbazolium betaine (M) and its protonated form (Miti $)$ in their excited singlet states $\left(\mathrm{S}_{1}\right)$ was studied using picosecond laser flash photolysis. From the time-resolved transient spectra direct evidence was obtained for the deprotonation of trans- $-\mathrm{HLI}^{+}$in the $S_{1}$ state, competing with isomerization.
\end{abstract}

\section{Introduction}

The photomulued trans/cis isomerization is one of the most important fundamental processes in photochemical and photobiological reactions. and many investigations have been carried out in order to elucidate its mechanism. Recently, direct observation of the isomerization process in the singlet excited state has become possible owing to the development of pieosecond laser speciroscopy. Up to now, however, the systems studied with this method are rather limited. The picosecond studies have been concentrated on silbenes [1] and rhodopsins [2]. A picosecond laser photolysis study of the singlet excited state of thioindigo has also been reported recently [3.4].

On the other hand. a detailed study of the photoisomerization has been mate in the merocyanine dye of stilbazolium betaine ( $M$ ) type by making the measurements of the reaction yields with the stationary method $[5]$. According to this study. $M_{\text {trans }}$ is more stable than $M_{\text {cis }}$ ind does not show photoisomerization. However, the $\mathrm{O}$-protunated form $\left(\mathrm{MH}^{+}\right)$readily

- Present address: Institute of Molecular Science, Okazaki \$44. Japan.

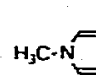<smiles>[C]1C=CC=C1</smiles>
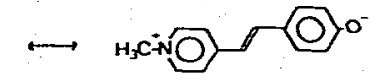

$\mathbf{M}$

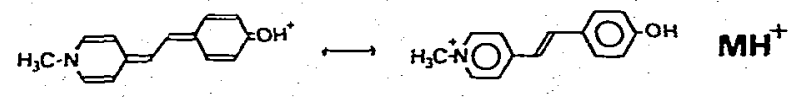

undergoes trans/cis photoisomerization. Owing to this peculiar behavior in aqueous solution, $M$ shows the following reaction cycle:

$$
\mathrm{M}_{\text {trans }} \stackrel{+\mathrm{H}^{+{ }_{1}} \rightleftharpoons}{\rightleftharpoons} \mathrm{MH}_{\mathrm{trans}}^{+} \stackrel{h \nu}{\rightleftharpoons} \mathrm{MH}_{\text {cis }}^{+} \stackrel{-\mathrm{H}^{+}}{\rightleftharpoons} \mathrm{M}_{\text {cis }} \rightarrow \mathrm{M}_{\text {trans }}
$$

This reaction cycle is interesting as a chemical model related to the molecular mechanism of some important photobiological phenomena such as the lightdriven proton pump of Halobacterium halobium or the process of vision [5].

Because of its interesting properties of intramolecular charge transfer as well as protonation coupled with photoisomerization, we have examined this system by means of picosecond laser photolysis in order to observe directly the relevant processes and to elucidate the details of the reaction mechanism. 


\section{Experimental}

The merocyanine dye, 4'-hydroxy-1-methylstilbazolium betaine $\left(\mathrm{MH}^{+}\right)$, was the same sample as used before [4]. Deionized and distilled water was distilled again with a non-boiling type distillation apparatus made of quartz. $98 \% \mathrm{H}_{2} \mathrm{SO}_{4}$ and $\mathrm{pH} 6$ as well as pH 10 buffer solutions (Puffer-Titrisol Merk) were used to control the $p H$ of the sample solutions.

Absorption and fluorescence spectra were measured by using respectively a JASCO UVIDEC-1 type spectrophotometer and an Aminco-Bowman spectrophotofluorometer.

Picosecond time-resolved transient absorption spectra and fluorescence decay curves were measured by means of a microcomputer-controlled picosecond laser photolysis system with a repetitive modelocked $\mathrm{Nd}^{3+}$ :YAG laser as the excitation source, details of which were reported elsewhere $[6,7]$.

In order to minimize the permanent trans/cis conversion in the observed volume, the solution in the sample cell was stirred after every shot. The sample solutions were deoxygenated by irrigating them with a purified nitrogen gas stream. Measurements were carried out at room temperature $\left(26 \pm 1{ }^{\circ} \mathrm{C}\right)$.

\section{Results and discussion}

The absorption spectra of $\mathrm{M}$ and $\mathrm{MH}^{+}$are shown in fig. la. At $p H \geqslant 10$, the concentration of the protonated species is negligible and the solution of $M$ is photochemically stable. At $p H \leqslant 6$, only the $\mathrm{MH}^{+}$ species is present in the ground state. $\mathrm{MH}^{+}$shows trans/cis photoisomerization and irradiation of $\mathrm{MH}_{\text {trans }}^{+}$leads to a trans/cis mixture. The broken curve in fig. la shows the spectra of the cis form calculated by Steiner et al. [5]. The fluorescence spectra are also shown in fig. $1 \mathrm{~b}$. The absorption spectrum of the merocyanine in $5 \% \mathrm{H}_{2} \mathrm{SO}_{4}$ solution was the same as that obtained at $\mathrm{pH} 6$.

Time-resolved transient absorption spectra of $\mathrm{MH}_{\text {trans }}^{+}$in buffer solution of $p H 6$ are shown in fig. 2a. Fig. 3a shows semilogarithmic plots of transient absorbance at 430 and $700 \mathrm{~nm}$. The absorbance at $430 \mathrm{~nm}$ shows a two-component decay. The decay time of the fast component estimated by subtracting the slow component from the observed decay curve
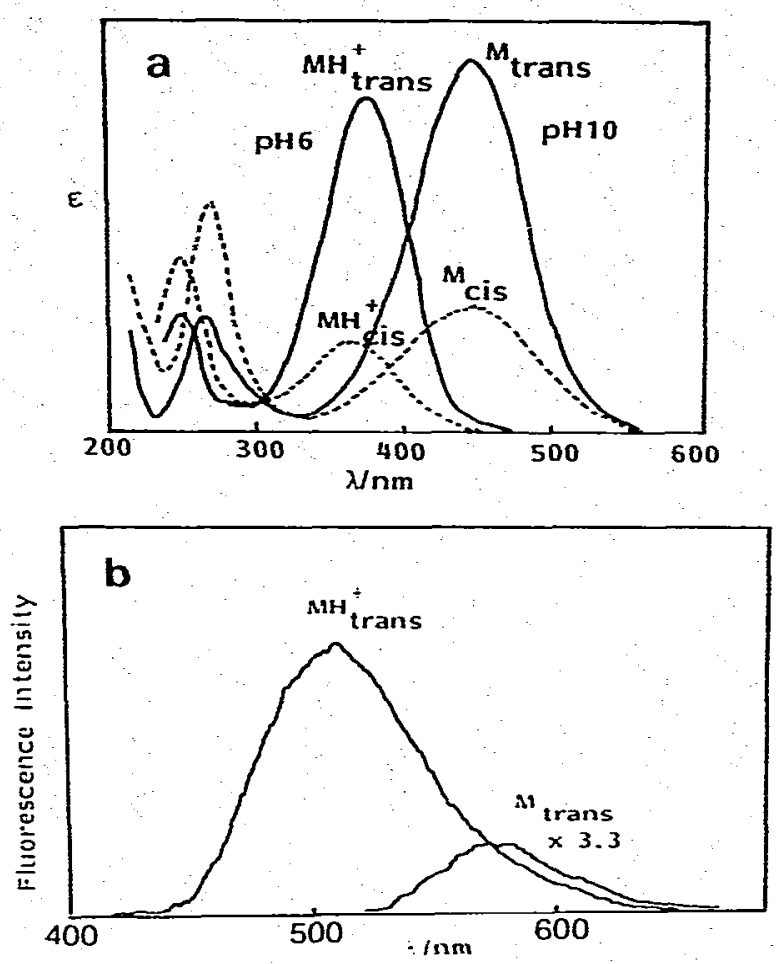

Fig. 1. (a) Ground-state absorption spectra of $\mathbf{M}$ and $\mathrm{MH}^{+}$in aqueous solution. (b) Fluorescence spectra of $\mathrm{M}(p H 10)$ and $\mathrm{MiH}^{+}(\mathrm{pH}$ 6) in aqueous solution.

was very short: this corresponds to the very short fluorescence lifetime observed for the same solution by means of a streak camera (see fig. $3 b$ ). These results indicate that the transient absorption at $430 \mathrm{~nm}$ which shows rapid decay is due to the $S_{n} \leftarrow S_{1}$ transition of $\mathrm{MH}_{\text {trans }}^{+}$

The absorbance in fig. 2a shows negative values around $500 \mathrm{~nm}$. This wavelength region coincides with that of the fluorescence band of $\mathrm{MH}_{\mathrm{trans}}^{+}$. which indicates that the monitoring light pulse of the picosecond continuum is a little amplified by the induced emission from the $\mathrm{S}_{1}$ state of $\mathrm{MH}_{\mathrm{trans}}^{+}$in the wavelength region of fluorescence. On the other hand, the strong absorption band around $700 \mathrm{~nm}$ is typical of the hydrated electron $\left(\mathrm{e}_{\text {sol }}^{-}\right)[\mathrm{S}]$, which seems to be produced by a biphotonic process.

In the time-resolved spectra in fig. $2 a$ an absorption peak is observed at $440 \mathrm{~nm}$ after rapid decay of the fast component at $430 \mathrm{~nm}$. It might be possible 

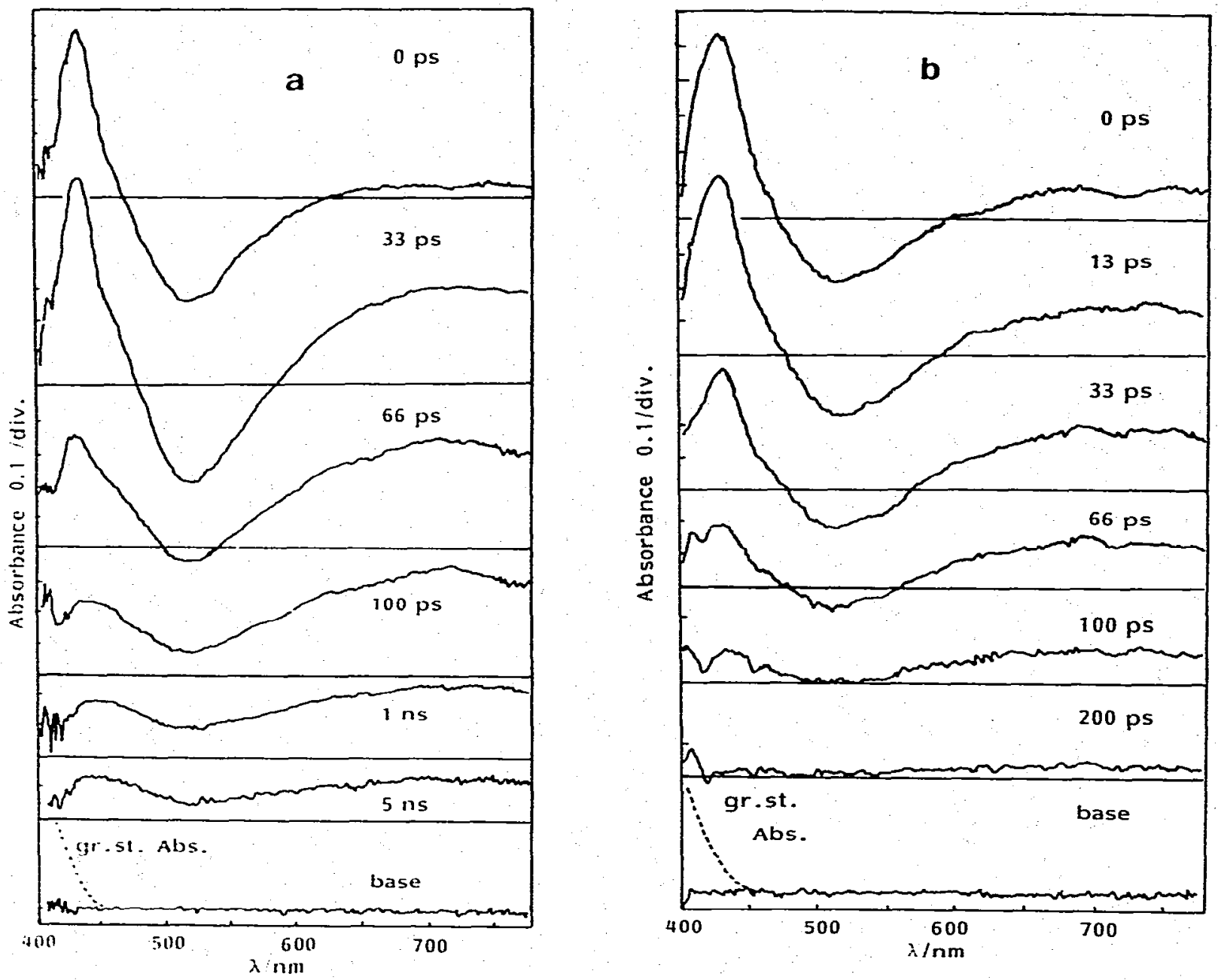

Hig. 2. (a) Time-sesolved transicnt absorption spectra of $\mathrm{MH}^{+}$in aqueous solution (pH 6). Concentration of $\mathrm{MH}^{+}: 7 \times 10^{-5} \mathrm{M}^{-}$(b) Time-resolved iransient absorption spectra of $\mathrm{MlH}^{+}$in $5 \% 1 \mathrm{H}_{2} \mathrm{SO}_{4}$ aqueous solution. Concentration of $\mathrm{MH}^{+}: 4 \times 10^{-5} \mathrm{M}^{-}$

that the absorption around $440 \mathrm{~nm}$ is due to a radical species such as $\mathrm{MH}^{2+}$ produced by electron photoejection from $\mathrm{MH}^{+}$. To check this possibility we have examined how the transient absorbance, detected with a delay time of $100 \mathrm{ps}$, at $440 \mathrm{~nm}$ and $700 \mathrm{~nm}$ depends on the excitation pulse intensity. We found that the species absorbing $440 \mathrm{~nm}$ radiation is produced by a monophotonic process whereas a biphotonic process is responsible for the formation of the species absorbing $700 \mathrm{~nm}$ radiation. Thus one may conclude that the absorption band at $440 \mathrm{~nm}$ is not due to the radical species $\mathrm{MH}^{2}+$.

On the other hand, the peak wavelength of this absorption band coincides with that of the ground-state absorption band of $M_{\text {trans }}$. This result strongly indicates the occurrence of protolytic dissociation of the excited $\mathrm{MH}_{\text {trans }}^{+}$in competition with isomerization. In fact such a process is to be expected, since $\mathrm{MH}^{+}$ has a $p K^{* *}$ of $\approx 1.9$ in the $S_{1}$ state $[9,10]$, indicating that the molecule is a much stronger acid in the ex- 

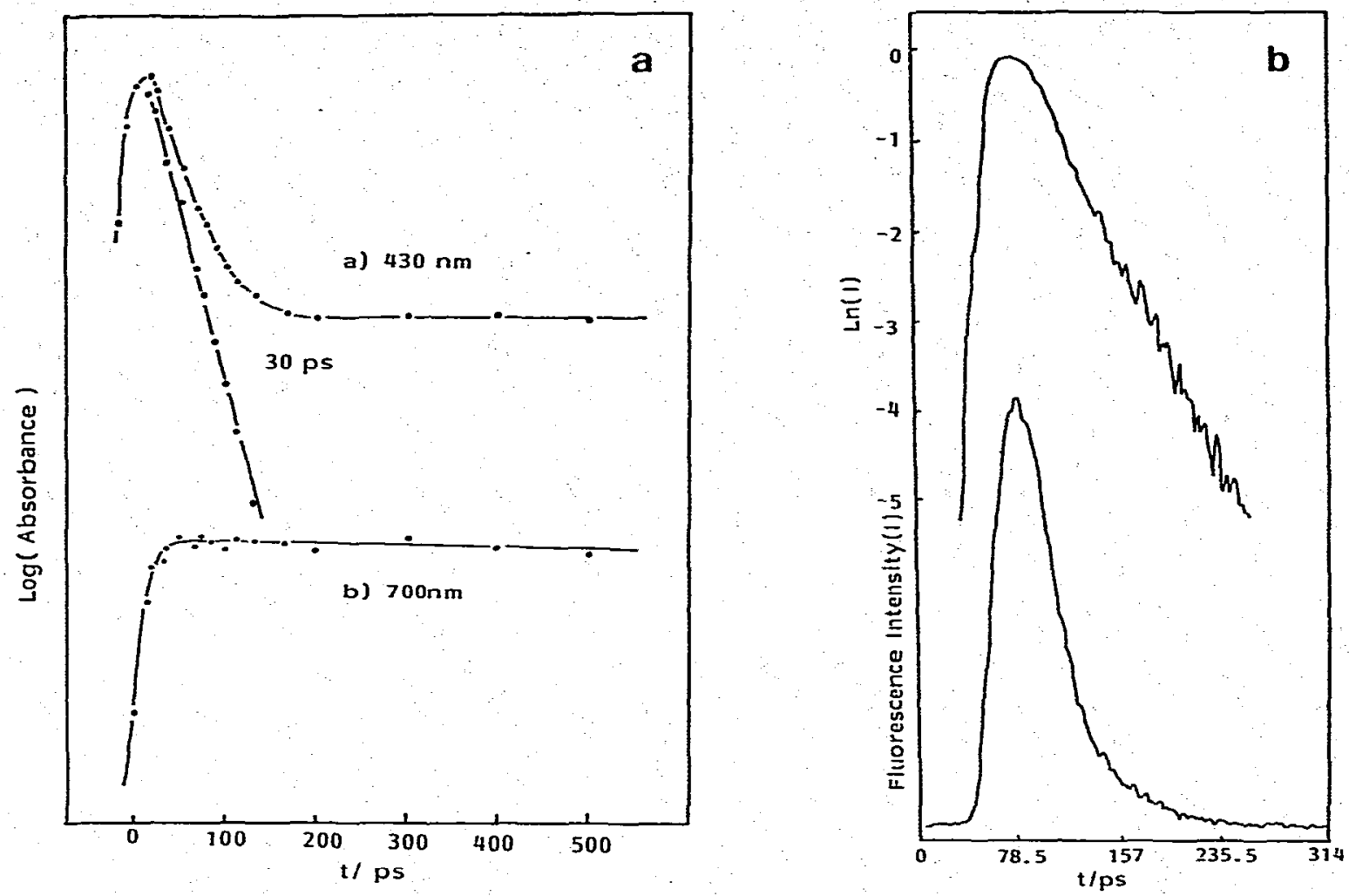

Fig. 3. (a) Semilogarithmic plot of transient absorbance of $\mathrm{MH}^{+}$in aqueous solution ( $p H$ 6). (b) Fluorescence decay curve of $\mathrm{MH}^{+}$ in aqueous solution $(\mathrm{pH}$ 6).

cited state than in the ground state $\left(p K_{\mathrm{G}}=8.54\right.$ at $\left.25^{\circ} \mathrm{C}[9]\right)$.

If * $\mathrm{MH}_{\text {trans }}^{+}$undergoes deprotonation, fluorescence from ${ }^{*} M_{\text {trans }}$ should be observable even if no $M_{\text {trans }}$ exists at $p H 6$ in the ground state. However, the yield of $\mathrm{M}_{\text {trans }}$ is apparently not very large, and since its fluorescence yield is quite small (cf. fig. $1 \mathrm{~b}$ ), the detection of the ${ }^{*} M_{\text {trans }}$ fluorescence will be difficult in the presence of the strong $\mathrm{MH}^{+}$fluorescence.

We made a comparative study of the behavior of * $\mathrm{MH}_{\text {trans }}^{+}$in solutions buffered at $\mathrm{pH} 6$ and solutions with $5 \% \mathrm{H}_{2} \mathrm{SO}_{4}$. We found that in such a strongly acidic solution the fluorescence yield was higher by $\approx 20 \%$ and the same applicd to the quantum yield $\phi_{\mathrm{tc}}$ of trans $\rightarrow$ cis photoisomerization. On the other hand a slight shift in the photostationary state towards a greater cis fraction $\neq$ when irradiated at $365 \mathrm{~nm}$, indicated that $\phi_{c t}$ did not change to a comparable extent. These findings altogether provide convincing evidence that deprotonation of * $\mathrm{MH}_{\text {trans }}^{+}$is suppressed in the strongly acidic solution.

It is of interest to re-examine the quantum yield data obtained by Steiner et al. [5] in the light of these results. It was suggested that the photoisomerization of $\mathrm{MH}_{\mathrm{trans}}^{+}$to $\mathrm{MH}_{\mathrm{cis}}^{+}$and vice versa proceeds via a common intermediate $X$. Thus the photochemical quantum yields may be decomposed as follows:

* It should be noted here that in $5 \% \mathrm{H}_{2} \mathrm{SO}_{4}$ solution the thermal back isomerization $\mathrm{MH}_{\text {cis }}^{+} \rightarrow \mathrm{MH}_{\text {trans }}^{+}$occurs - obviously acid catalyzed - at an appreciable rate (half conversion time being $\approx 2-3 \mathrm{~h}$ at $25^{\circ} \mathrm{C}$ ). 
$\phi_{\mathrm{tc}}=\phi_{\mathrm{IX}} \phi_{\mathrm{Xc}}$

$\phi_{\mathrm{ct}}=\phi_{\mathrm{cX}} \phi_{\mathrm{Xt}}$.

with the underlying assumption

$\phi_{\mathrm{Xc}}+\phi_{\mathrm{Xt}}=\mathrm{I}$.

On the other hand. $\phi_{\mathrm{c}} \mathrm{x}$ is generally assumed to equal 1. so that

$\phi_{\mathrm{Xt}}=\phi_{\mathrm{ct}}$

and combining (1). (3) and (4):

$\phi_{i x}=\phi_{t c} /\left(1-\phi_{c t}\right)$.

With $\phi_{t c}=0.33$ and $\phi_{c t}=0.57$ we obtain $\phi_{t X}=0.77$. In other words. 23\% of * $\mathrm{MH}_{\text {trans }}^{+}$disappear through other chamnels than isomerization. This figure compares well with the increase of $\phi_{f}$ and $\phi_{t c}$ upon acidification of the solution. meaning that deprotonation of ${ }^{*} \mathrm{MH}_{\text {trans }}^{+}$in weakly acid solution is the main side channel of deactivation besides isomerization.

Assuming that dissociation of ${ }^{*} \mathrm{MH}_{\text {trans }}^{+}$contributes $20 \%$ of the decay rate constant, which was obtained as $1.67 \times 10^{10} \mathrm{~s}^{-1}$ in weakly acid solutions [5], the rate constant of deprotonation would be $\approx 3 \times 10^{9} \mathrm{~s}^{-1}$. which compares well with cobserved values for molecules with strongly acidic excited states [11].

The results obtained with the $5 \% \mathrm{H}_{2} \mathrm{SO}_{4}$ solution of $\mathrm{MHH}_{\mathrm{trans}}^{+}$in the picosecond laser experiments are shown in fig. $2 b$. where the transient absorption spectra at early delay times from the excitation pulse are nearly the same as in fig. 2a. However, the absorption band at $440 \mathrm{~nm}$ observed at a delay time of $\approx 100 \mathrm{ps}$ in the case of the solution with $\mathrm{pH} 6$ cannot be recognized for this strongly acidic solution. Furthermore. $a t \approx 200 \mathrm{ps}$. not only the $430 \mathrm{~nm}$ band but also the ahsorption due to e solv ean be hardly observed.

The rapid disappearance of $e^{-}$solv absorption in strongly acidic solution may be ascribed to the reaction of $\mathrm{e}^{-}$solv with high concentration hydronium ions. The decay of the transient absorption due to $c_{\text {solv }}$ in $5 \% \mathrm{H}_{2} \mathrm{SO}_{4}$ solution was exponential, and the decay time was obtained to be $\approx 70 \mathrm{ps}$.

It should be noted here that. in strongly acidic solution. after the rapid decay of the $S_{n}-S_{1}$ absorption band of $\mathrm{MH}_{\mathrm{trans}}^{+}$and that of $\mathrm{e}_{\text {solv }}^{-}$, no absorption band due to long-lived excited species can be recognized in the visible region. No such absorption band can be recognized also in weakly acidic solution except for the absorption bands due to the transiently produced $M_{\text {trans }}$ and $e_{\text {solv }}^{-}$. These results support the mechanism of direct trans/cis photoisomerization from the $S_{1}$ state assumed in the previous report in analogy to the case of stilbene or stilbazoles [5].

We have examined also the excited state of $M_{\text {trans }}$ by means of picosecond laser photolysis method. Although we observed a fluorescence decay curve similar to that of $\mathrm{MH}_{\text {trans }}^{+}$by means of a streak camera, no appreciable transient absorption band except for that of $\mathrm{e}_{\text {solv }}^{-}$was detected in the measurable wavelength region $(500-800 \mathrm{~nm})$.

From the fact that $M_{\text {trans }}$ does not show photoisomerization and that the fluorescence radiative transition probability obtained from the fluorescence quant um yield and lifetime is considerably smaller than the value calculated from the absorption band, the following two alternative mechanisms have been proposed [5] for the behavior of excited $M_{\text {trans }}:$ (a) the singlet state $S_{1}$ reached upon excitation relaxes very rapidly to a lower singlet state $S_{1}^{\prime}$ with a smaller fluorescence transition probability; and (b) vibrational relaxation in $S_{1}$ state competes with an internal conversion to a singlet state $S_{1}^{\prime \prime}$ (situated above the zero-point vibrational state of $S_{1}$ ) which is very efficiently deactivated non-radiatively. The present result of picosecond laser photolysis seems to indicate that the $S_{n} \leftarrow S_{1}$ band of the fluorescent state cannot be detected due to the very small concentration of $S_{1}$ state. i.e. mechanism (b). However, it might also be

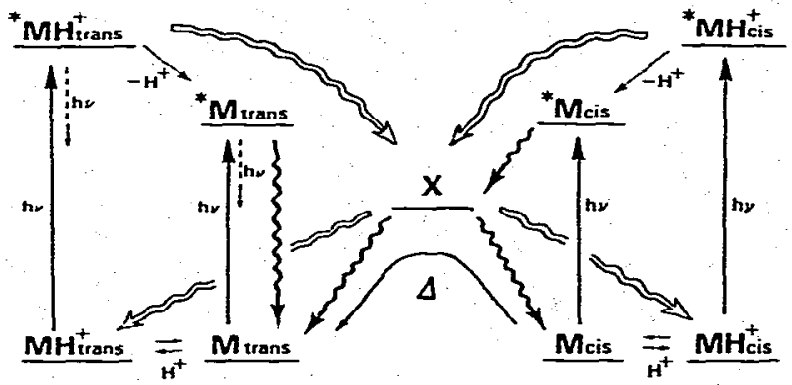

Fig. 4. A schematic diagram for the photochemical processes of $\mathrm{M}$ and $\mathrm{MH}^{+}$. At $p H \mathrm{6}$, the distribution of the decay of various species over their respective decay channels is as follows. $* \mathrm{MH}_{\text {trans }}^{+} \rightarrow \mathrm{MH}_{\text {trans }}^{+}+h \nu: 1.5 \%, * \mathrm{MH}_{\text {trans }}^{+} \stackrel{-\mathrm{H}^{+}}{\rightarrow} * \mathrm{M}_{\text {trans }}$ : $20 \%,{ }^{*} \mathrm{MHI}_{\text {trans }}^{+} \rightarrow \mathrm{X}: 77 \%,{ }^{*} \mathrm{MH}_{\text {cis }}^{+} \rightarrow \mathrm{X}: 100 \%, \mathrm{X} \rightarrow \mathrm{MH}_{\text {trans }}^{+}$ $57 \%, \mathrm{X} \rightarrow \mathrm{MH}_{\mathrm{cis}}^{+}: 43 \%$ : 
possible that the $S_{n} \leftarrow S_{1}$ band is hidden in the region of the strong ground-state absorption band of $\mathrm{M}_{\text {trans }}$ -

Summarizing the above results together with the previous ones, the photochemical processes of the present system may be represented schematically as indicated in fig. 4.

\section{Acknowledgement}

The present work was supported by Grant-in-Aid for Special Project Research on Photobiology from the Japanese Ministry of Education, Science and Culture to NM.

\section{References}

[1] B.I. Greene, R.M. Hochstrasser and R.B. Weisman, Chem. Phys. Letters 62 (1979) 427;

M. Sumitani, N. Nakashima and K. Yoshihara, Chem. Phys. Letters 68 (1979) 255:

[2] $R$. Callender, in: Biological events probed by ultrafast laser spectroscopy, ed. R.R. Alfano (Academic Press, New York, 1982) p. 239;
K.E. Peters and N. Leontis, in: Biological events probed by ultrafast laser spectroscopy, ed. R.R. Alfano (Academic Press, New York, 1982) p. 259;

T.G. Ebrey, in: Biological events probed by ultrafast laser spectroscopy, ed. R.R. Alfano (Academic Press. New York, 1982) p. 271.

[3] S.A. Krysanov and M.V. Alfimov, Chem. Phys. Letters 76 (1980) 221;82 (1981) 51.

[4] N. Mataga, Y. Maeda and T. Okada, to be published.

[5] Y. Steiner, M.H. Abdel-Kader, P. Fiseher and H.E.A. Kramer, J. Am. Chem. Soc. 100 (1978) 3190.

[6] H. Masuhara, N. Ikeda, H. Miyasaka and N. Mataga, J. Spectry. Soc. Japan 31 (1982) 19.

[7] H. Miyasaka, H. Masuhara and N. Mataga, Laser Chem., submitted for publication.

[81 J.W. Boag and E.J. Hart, Nature 197 (1963) 45; M. Imamura, A. Kira and S. Arai, in: Kagakusosetsu, No. 24, Chem. Soc. Japan (Japan Scientific Societies Press, 1979) p. 181.

[9] J.E. Kuder and D. Wychik, Chem. Phys. Letters 24 (1974) 69.

[10] M.H. Abdel-Kader, Thesis, Stuttgart University (1978) p. 114.

[11] M. Hauser, H.-P. Haar and U.K.A. Klein, Ber. Bunsenges: Physik. Chem 81 (1977) 27;

F. Hafner, J. Wörner, U. Steiner and M. Hauser, Chem. Phys. Letters 73 (1980) 139;

H. Shizuka, K. Tsutsumi, H. Takeuchi and 1. Tanaka, Chem. Phys. Letters 62 (i979) 408. 